\title{
LA EPIDEMIA DE FIEBRE AMARILLA DE 1800 Y SU IMPACTO EN LA \\ CARLOTA, CAPITAL DE LAS NUEVAS POBLACIONES DE ANDALUCÍA ${ }^{1}$ \\ ADOLFO HAMER-FLORES \\ UNIVERSIDAD LOYOLA ANDALUCÍA
}

\begin{abstract}
RESUMEN: Las sociedades de finales del Antiguo Régimen se vieron amenazadas por diferentes enfermedades, siendo la fiebre amarilla una de las más temidas por su letalidad. Este trabajo expone una panorámica general de la epidemia de fiebre amarilla de 1800 en España, dedicando una especial atención al caso de La Carlota (Córdoba). Esta enfermedad nunca había penetrado con anterioridad hasta un lugar tan alejado de la costa española.
\end{abstract}

PALABRAS CLAVE: Nuevas Poblaciones de Andalucía, La Carlota, epidemia, fiebre amarilla, 1800.

\section{THE YELLOW FEVER EPIDEMIC OF 1800 AND ITS IMPACT ON LA CARLOTA, CAPITAL OF THE NEW SETTLEMENTS OF ANDALUSIA}

\begin{abstract}
The societies in the Late Old Regime were threatened by different diseases, the yellow fever being one of the most feared because their lethality. This paper shows a general overview of the epidemic of yellow fever of 1800 in Spain. It pays a special attention to the case of La Carlota (Córdoba). This disease had never penetrated before to a place so far from the Spanish coast.
\end{abstract}

KEYWORDS: New Settlements of Andalusia, La Carlota, epidemic, yellow fever, 1800.

Recibido: 10-02-2018/Aceptado: 07/04/2018

\footnotetext{
${ }^{1}$ El presente trabajo ha sido realizado en el contexto de nuestra labor científica como miembro del Grupo de Investigación HISALEM (Historia Social de la Administración Local en la Época Moderna), código HUM-121, perteneciente al Plan Andaluz de Investigación de la Junta de Andalucía.
} 


\section{Introducción}

Los episodios epidémicos constituyeron una realidad habitual en la España del Antiguo Régimen, estando presentes desde brotes que podemos considerar menores, como los de gripe ${ }^{2}$, hasta otros de gran impacto demográfico y social que interrumpían el devenir cotidiano. Los avances médicos y el conocimiento de esas enfermedades, que han ido haciendo posible su control hasta poder considerar casi erradicadas a algunas de ellas, aún estaban en ciernes en la época que aquí nos ocupa. Una vulnerabilidad que se hacía más patente ante enfermedades no endémicas, y que eran transportadas de unos lugares a otros por comerciantes y viajeros. Este fue el caso de la fiebre amarilla que, procedente de América, llegaría, como en otras ocasiones anteriores, al puerto de Cádiz en los últimos momentos del siglo XVIII. Ni la habitual cuarentena a la que eran sometidos los barcos pudo impedir su amplia difusión o que en esta ocasión tuviese un impacto mucho mayor.

A partir de este primer episodio, la enfermedad se hizo habitual en numerosas ciudades andaluzas y del litoral murciano y alicantino, e incluso se extendió en $1821^{3}$ y $1870^{4}$ más al norte, siendo responsable de miles de fallecimientos en Barcelona. De este modo, las epidemias de fiebre amarilla y de cólera morbo fueron las causantes directas de la implementación de los preceptos higienistas en España y de una mayor concienciación, tanto en sectores médicos como políticos, por la salubridad, la sanidad y la organización sanitaria. A modo de ejemplo, la enfermedad que aquí nos ocupa tendría un papel de primer orden en el primer intento por establecer una ley general de sanidad para toda España durante el

\footnotetext{
2 Aunque cada año enferman unos 500 millones de personas, solo de tres a cinco millones evolucionan a formas graves, falleciendo de ellas entre 250 y 500 mil cada año. No obstante, tampoco esta enfermedad está exenta de episodios pandémicos como, por ejemplo, la "gripe española" de 1818-1919, que dejó 40 millones de muertos, la gripe asiática de 1957, con sus dos millones de muertes, o la gripe de Hong Kong de 1968, con su millón de fallecidos (ROMERO CABELLO, Raúl: Microbiología y parasitología bumana. Bases etiológicas de las enfermedades infecciosas y parasitarias, México, Editorial Médica Panamericana, $3^{\mathrm{a}}$ ed., 2007, p. 489).

${ }^{3}$ En el caso de la epidemia de fiebre amarilla de 1821 en Barcelona, además de uno de los primeros testimonios casi coetáneos (Manifiesto acerca del origen y propagación de la calentura que ha reinado en Barcelona en el año 1821, presentado al Augusto Congreso Nacional por una reunión libre me médicos estrangeros y nacionales, Madrid, Imprenta de Albán y Compañía, 1822), disponemos de un par de trabajos fundamentales: ORTIZ GARCÍA, José Antonio: "Autoridad e imagen de la epidemia. La fiebre amarilla en la Barcelona del siglo XIX”, Potestas, 11 (2017), pp. 93-110; y GASPAR GARCÍA, Ma Dolores: "La epidemia de fiebre amarilla que asoló Barcelona en 1821, a través del contenido del manuscrito 156 de la Biblioteca Universitaria de Barcelona", Gimbernat: revista catalana d'història de la medicina i de la ciencia, 18 (1992), pp. 65-72.

4 Para el brote epidémico de 1870 contamos con trabajos que nos aportan información acerca de algunos de los escenarios más afectados como, por ejemplo, Barcelona (MENDOZA, Antonio: Memoria histórico-científica sobre la epidemia de fiebre amarilla sufrida en Barcelona en 1870, Barcelona, Establecimiento Tipográfico de Jaume Jepús, 1872; ORTIZ GARCÍA, José Antonio: Op. cit.) y Alicante (SEGUÍ MARCO, Guillermo: "La epidemia de fiebre amarilla de 1870 en Alicante”, Anales de la Universidad de Alicante. Historia Contemporánea, 2 (1983), pp. 109-134).
} 
Trienio Liberal (el Proyecto de Código Sanitario de 30 de abril de 1822), al igual que en la elaboración del primer código sanitario español, la Ley Orgánica de Sanidad de $1855^{5}$. Todo ello justifica sobradamente la necesidad de seguir ahondando en el conocimiento de los primeros brotes epidémicos de fiebre amarilla en nuestro país.

En este trabajo nos centraremos, por tanto, en la primera de esas grandes epidemias de fiebre amarilla, la de 1800, analizando el caso de la localidad más alejada de la costa gaditana en la que se hicieron presentes sus virulentas características. Nos referimos a La Carlota $^{6}$, capital de las Nuevas Poblaciones de Andalucía, una de las dos capitales de la conocida como Intendencia de las Nuevas Poblaciones de Sierra Morena y Andalucía; fundada solo unas décadas antes. Hasta la fecha solo contamos con referencias puntales a esta realidad y con alguna cifra acerca del número de óbitos que provocó entre sus habitantes, de ahí que consideremos de utilidad ofrecer un análisis más detallado del desarrollo de la epidemia en ella, así como de los efectos sociopolíticos que desencadenó, pues alteró notablemente la tranquilidad institucional y de gobierno en las referidas Nuevas Poblaciones de Andalucía durante varios años.

Un objetivo para el que nos apoyaremos en fuentes de archivo, en buena medida inéditas, así como en testimonios impresos coetáneos y en las investigaciones generadas por la comunidad científica durante las últimas décadas. Aspiramos de este modo a mejorar de algún modo el nivel de conocimiento actual de los efectos que tuvo en nuestro país la epidemia de fiebre amarilla de 1800 .

\section{Las epidemias de fiebre amarilla de $\mathbf{1 8 0 0}$}

Desde comienzos del siglo XVIII, los contactos entre las áreas endémicas de la fiebre amarilla $\mathrm{a}^{7}$ los puertos europeos constituyeron una realidad cada vez más frecuente; de ahí el creciente impacto en el espacio europeo de brotes epidémicos de esta enfermedad. Durante esta centuria, hizo acto de presencia en Lisboa en 1723, en Cádiz en 1730 y 1733, en Málaga en 1741 y de nuevo en Cádiz en 1800. Aun así, el periodo de mayor repercusión en Europa, sin duda, fue la primera mitad del siglo XIX, tanto por la gravedad de las epidemias como

\footnotetext{
${ }^{5}$ FERIA LORENZO, Diego José: “La primera regulación sanitaria española: la Ley Orgánica de 1855”, Revista de História das Ideias, 33 (2012), pp. 217-239.

${ }^{6}$ En la actualidad, La Carlota es un municipio enclavado en la provincia de Córdoba.

${ }^{7}$ MENDOZA, José: Historia del carácter, síntomas y método curativo y preservativo de la enfermedad contagiosa que se experimenta en la actualidad en esta ciudad de Córdoba, Córdoba, s.i., 1804, p. 3. Esta enfermedad recibe en los círculos médicos de la época diversas denominaciones, indicándonos Mendoza que se trataba de tifus icterodes, al cual "vulgarmente se le da el [nombre] de fiebre amarilla y vómito prieto [o negro]".
} 
por su enorme difusión geográfica ${ }^{8}$.

La constatación de casos puntuales de enfermos no implicaba un riesgo forzoso para la población. El desencadenamiento de un brote epidémico de fiebre amarilla necesitaba del concurso de diversos factores: el agente causal, la existencia de mosquitos vectores y la presencia de seres humanos susceptibles de contraer la enfermedad. Un hecho que venía a dificultar, en gran medida, su difusión; la cual solo era posible en unas condiciones muy determinadas. El vector o agente causal ${ }^{9}$ solo se desarrolla en un medio tropical o subtropical, siempre en un contexto de temperaturas altas y en un medio lacustre ${ }^{10}$. Por debajo de los $25^{\circ} \mathrm{C}$ su actividad se reduce, y con temperaturas inferiores a los $17^{\circ} \mathrm{C}$ casi desaparece. La fiebre amarilla, por tanto, es una enfermedad de ámbito eminentemente geográfico: la franja tropical y subtropical del planeta. Por otra parte, en caso de transmisión de hombre a hombre, el periodo de riesgo no suele superar el par de días, con independencia de la fuerza del ataque. Una circunstancia que nos muestra que la expansión de la fiebre amarilla tiene un marcado carácter local ${ }^{11}$, lo cual no fue óbice para que, en momentos puntuales, con las condiciones climáticas adecuadas, se mostrase con toda su virulencia en el viejo continente.

\subsection{Origen de la enfermedad}

A pesar de que se formularon diversas hipótesis acerca del posible foco originario desde el que irradió la epidemia de fiebre amarilla de $1800^{12}$, la afirmación de Juan Manuel de Aréjula acerca de que la enfermedad fue traída a las costas gaditanas por un barco procedente del área caribeña, arribado a su puerto el seis de julio de ese mismo año, ha sido confirmada en análisis históricos posteriores ${ }^{13}$. Pero ¿por qué en Cádiz? Con toda probabilidad debido a que se trataba del puerto por excelencia de la Península Ibérica, aquel

\footnotetext{
8 CARRILLO, Juan L. y GARCÍA-BALLESTER, Luis: Enfermedady sociedad en la Málaga de los siglos XVIII y XIX. I. La fiebre amarilla (1741-1821), Málaga, Universidad de Málaga, 1980, p. 39.

${ }^{9}$ La fiebre amarilla tiene como agente causal un virus del grupo B de los arbovirus, y prototipo del grupo taxonómico de los flavovirus.

${ }^{10}$ IGLESIAS RODRÍGUEZ, Juan José: La epidemia gaditana de fiebre amarilla de 1800 (el caso de Puerto Real), Cádiz, Diputación Provincial de Cádiz, 1987, p. 111.

11 ARJONA CASTRO, Antonio: La población de Córdoba en el siglo XIX. Sanidad y crisis demográfica en la Córdoba decimonónica, Córdoba, Universidad de Córdoba, 1979, p. 26.

${ }^{12}$ La bibliografía que ha abordado este episodio epidémico es muy abundante. Además de los trabajos referenciados en otras notas del presente trabajo, consideramos imprescindible mencionar aquí un par de trabajos centrados en el área gaditana (IGLESIAS RODRÍGUEZ, Juan José: Una ciudad mercantil en el siglo XV III: El Puerto de Santa María, Sevilla, Muñoz Moya y Montreveta, 1991; y RODRÍGUEZ CARRIÓN, José: Jerez, 1800: epidemia de fiebre amarilla, Jerez de la Frontera, Centro de Estudios Históricos Jerezanos, 1980) y otro sobre la presencia de la enfermedad en Sevilla (HERMOSILLA MOLINA, Antonio: Epidemia de fiebre amarilla en Sevilla en el año 1800, Sevilla, Talleres Gráficos Oiga, 1978).

13 IGLESIAS RODRÍGUEZ, Juan José: Op. cit., pp. 112 y 116.
} 
que hasta hacía muy pocos años había disfrutado del privilegio del monopolio del comercio con América ${ }^{14}$. Los contactos por mar con áreas donde la enfermedad era endémica eran mucho más frecuentes en esta ciudad, lo que incrementaba notablemente las posibilidades de su trasmisión.

Aún así, la propagación de la epidemia no hubiera sido posible sin unos condicionantes muy específicos que se dieron en aquel momento. En este sentido, nos consta que el invierno de los años 1799-1800 había sido muy húmedo, con lluvias abundantes hasta finales de mayo; con una llegada brusca de las altas temperaturas ${ }^{15}$. Tanto es así que la temperatura media de 1800 superaría en casi seis grados a la del año anterior, lo que tuvo como consecuencia un verano seco y caluroso. Un contexto proclive a la difusión de la enfermedad que, seguramente, también se vio ayudado por la existencia de aguas estancadas ${ }^{16}$.

Del 10 al 15 de agosto de 1800 se manifiesta la enfermedad en Cádiz. Ataca en primer lugar al barrio de Santa María, situado al sureste de la ciudad ${ }^{17}$, y habitado sobre todo por marineros y empleados de la aduana ${ }^{18}$; pero se extenderá muy rápidamente. En un parte médico que el doctor Alfonso de María pasó a la Junta de Sanidad de Puerto Real, con fecha de 12 de septiembre, éste afirmaba que "desde el día 20 del próximo agosto oía hablar con inquietud de una enfermedad que con estrago progresaba en la ciudad de Cádiz [...]"19. Entre los meses de agosto y octubre fallecerían en Cádiz 7.387 personas, lo que supuso un 10,33\% de su censo, mientras otro 19,58\% del total huyó de la ciudad, contribuyendo con mucha probabilidad a su rápida difusión. Por ejemplo, a fines de julio la fiebre amarilla ya se había declarado en Sevilla, donde enfermó casi el $95 \%$ de su población ${ }^{20}$ y se cobró la elevada cifra de 16.685 vidas $^{21}$. Tan solo el 13,55\% de los gaditanos pudo esquivar la enfermedad, que sí padeció, aunque pudieron restablecerse, el restante $57,53 \%{ }^{22}$. Unas cifras que muestran el alto grado de impacto que esta epidemia tuvo en su área de influencia, al haber afectado al $67,86 \%$ de la población censada.

\footnotetext{
${ }^{14}$ ARJONA CASTRO, Antonio: Op. cit., p. 26.

15 BERTHE, Jean Nicolas: Précis historique de la maladie qui a règne dans l'Andalousie en 1800 (années 8 et 9 de la République française), Paris-Montpellier, chez Déterville-chez Renaud, 1802, p. 51.

${ }^{16}$ IGLESIAS RODRÍGUEZ, Juan José: Op. cit., pp. 112 y 116-118.

17 DE LA CRUZ BAHAMONDE, Nicolás: De Cádizy su comercio (Tomo XIII del Viaje de España, Francia e Italia), Cádiz, Universidad de Cádiz, 1997, pp. 247-250. Nicolás de la Cruz fue testigo presencial de la aparición de la enfermedad en el barrio de Santa María y de cómo se difundió por otras zonas de la ciudad.

18 ARÉJULA, José Manuel: Breve descripción de la fiebre amarilla padecida en Cádizy pueblos comarcanos en 1800, en Medina Sidonia en 1801, en Málaga en 1803, y en esta misma plaza y otras del reino en 1804, Madrid, Imprenta Real, 1806, p. 245; BERTHE, Jean Nicolas: Op. cit., p. 52.

19 IGLESIAS RODRÍGUEZ, Juan José: Op. cit., p. 125.

20 SUÁREZ FERNÁNDEZ, Luis: Del Antiguo al Nuevo Régimen: hasta la muerte de Fernando VII, Madrid, Ediciones Rialp, 1981, tomo XII, p. 8.

${ }^{21}$ IGLESIAS RODRÍGUEZ, Juan José: Op. cit., p. 31.

22 SUÁREZ FERNÁNDEZ, Luis: Op. cit., p. 8.
} 


\subsection{Diagnósticos y descripciones}

Conocer con exactitud qué dolencia o enfermedad aquejaba a un enfermo en la España de finales del Antiguo Régimen era una tarea muy compleja y, en no pocas ocasiones, alejada de las posibilidades reales de los facultativos. La mayor parte de los médicos se enfrentaban al hecho de establecer un diagnóstico con el insalvable inconveniente de una formación académica que no les permitía hacer un diagnóstico demasiado preciso. Ahora bien, no sería éste el único inconveniente que vino a dificultar la correcta identificación de la fiebre amarilla, sino el carácter intermitente de la enfermedad ${ }^{23}$ y el propio temor de estos profesionales a la reacción de la población si se declaraba su carácter epidémico. Como consecuencia, no faltaron en la época numerosas disputas en los círculos médicos que tuvieron como objeto de discusión el supuesto carácter contagioso de la enfermedad. Tampoco facilitaba mucho el acuerdo el hecho de que ésta confiere al que la sufre y sobrevive una inmunidad total y permanente ${ }^{24}$. Así pues, no debe extrañarnos que para el caso concreto de la epidemia en La Carlota, ésta nunca sea calificada en los documentos de archivo con términos que vayan más allá de "calenturas malignas o tabardillo"; el término de fiebre amarilla no era manejado por quienes hablaban de la epidemia ${ }^{25}$.

El periodo de incubación duraba de tres a seis días, y entre los síntomas destacaba la fiebre, la icteria (a causa de la cual los enfermos tomaban una tonalidad amarillenta), fuertes dolores de cabeza, dolor de estómago, vómitos frecuentes (que derivaban a la semana en vómitos negros, compuestos en gran medida de sangre oscura grumosa), sangrado por nariz y boca, palidez, sudores copiosos, fotofobia y convulsiones. La enfermedad dejaba como rastro particular una alta proporción de defunciones (que podía ir del 20 al 90\%) entre el quinto y el noveno día, bien por coma hepático o bien por insuficiencia suprarrenal o circulatoria.

Los enfermos recibían el tratamiento que el criterio particular de cada médico estimaba más eficaz. En cualquier caso, era común el evitar que estos ingiriesen alimentos sólidos, el aplicarles lavativas emolientes con vinagre y el darles frecuentemente agua, todo con objeto de evitar los vómitos y propiciar el sudor. No faltaban tampoco las habituales sangrías o el uso de quina donde se disponía de ella. En cualquier caso, el método más efectivo para prevenir el contagio en aquel entonces siempre fue la prevención ${ }^{26}$.

${ }^{23}$ HIELO SERRANO, Manuel y otros: Estudios especiales de la fiebre amarilla ó tifus icteroides, Barcelona, s.i., 191?, p. 24.

${ }^{24}$ CARRILLO, Juan L. y GARCÍA-BALLESTER, Luis: Op. cit., p. 33.

25 Archivo Histórico Nacional (en adelante A.H.N.), Fondos Contemporáneos, Gobernación, leg. 330, exp. 9.

${ }^{26}$ IGLESIAS RODRÍGUEZ, Juan José: Op. cit., pp. 118, 121 y 125. 


\section{Las repercusiones de la fiebre amarilla de 1800 en La Carlota, capital de las Nuevas}

\section{Poblaciones de Andalucía}

La población de La Carlota, capital de las Nuevas Poblaciones de Andalucía, atravesaba a finales del siglo XVIII una etapa de crecimiento demográfico limitado, a nivel casi de estancamiento, resultado evidente de los ajustes que se producirían tras el fortísimo crecimiento demográfico experimentado tras su fundación en 1768. Justo en esta coyuntura tan delicada, coincidiendo con los últimos meses de la centuria, hizo acto de presencia en ella una mortífera epidemia de fiebre amarilla. Su emplazamiento junto al camino real que unía Madrid con Cádiz facilitaba que diariamente la transitaran viajeros en uno u otro sentido. En los días que transcurrieron entre el 2 de septiembre y el 16 de noviembre de $1800^{27}$, esta nueva población experimentaría unos niveles de mortalidad catastrófica como no se recordaba desde las virulentas epidemias de paludismo de sus primeros años de existencia ${ }^{28}$. El pico de mortalidad registrado en ese año destaca sobremanera en el contexto de la década en el que se enmarca (Gráfico 1), y tuvo su reflejo en una leve caída de la natalidad en $1801^{29}$.

$\mathrm{Al}$ parecer, el introductor de la epidemia en el casco urbano de La Carlota fue un valenciano llamado Juan, del cual solo sabemos que "benía con un carro" y que murió el 2 de septiembre de 1800 en su Real Posada ${ }^{30}$. Aunque desconocemos cuál había sido el itinerario en su viaje, cabe suponer que acababa de llegar de algún lugar ya contagiado del limítrofe reino de Sevilla. En cualquier caso, la enfermedad pronto se propagaría entre los arrendadores, empleados e inquilinos de este edificio. Su difusión por el resto del núcleo urbano solo fue cuestión de días.

\footnotetext{
27 Archivo Parroquial de La Carlota (en adelante A.P.LC.), Libro $2^{\circ}$ de Entierros, ff. $1 \mathrm{r}-1 \mathrm{v}$.

28 Ver, entre otros, los trabajos de: MARTÍNEZ AGUILAR, Joaquín: "La Carlota: los primeros colonos y sus raíces”, en AVILÉS, Miguel y SENA, Guillermo (eds.): Carlos III y las Nuevas Poblaciones, Córdoba, Universidad de Córdoba y Seminario de Estudios Carolinenses, 1988, II, 303-363; y GARCÍA CANO, María Isabel: La colonización de Carlos III en Andalucía. Fuente Palmera 1768-1835, Córdoba, Diputación Provincial de Córdoba, 1982, pp. 53-55.

${ }^{29} \mathrm{El}$ número de nacimientos alcanzó las cifras previas a la epidemia de fiebre amarilla ya en 1802, contando incluso con un repunte al año siguiente. En lo que respecta a enlaces matrimoniales, también mostraron un levísimo incremento en 1801 y 1802. No obstante, consideramos que las condiciones climatológicas en los primeros años del siglo XIX, tan adversas que en el verano de 1804 casi no se recogió cosecha de cereal, actuaron como factor limitante en el número de nacimientos y enlaces matrimoniales, impidiendo apreciar los efectos que, a priori, cabía esperar tras la epidemia en estas series; en el caso de los nacimientos, cayeron en 1804 en más de un 25\% en relación al año anterior, y en el caso de los matrimonios, los enlaces en 1804 supusieron menos del 25\% de los realizados en 1801.

30 A.P.LC., Libro $1^{\circ}$ de Entierros, f. 295v. No obstante no sería esta la primera defunción anómala en este edificio, pues el 10 de agosto moriría allí "de repente" Alonso Muñoz, vecino de la cercana localidad de La Rambla (A.P.LC., Libro $1^{\circ}$ de Entierros, f. 295r.).
} 


\section{Gráfico 1}

Evolución de la natalidad y la mortalidad. La Carlota, 1796-1803

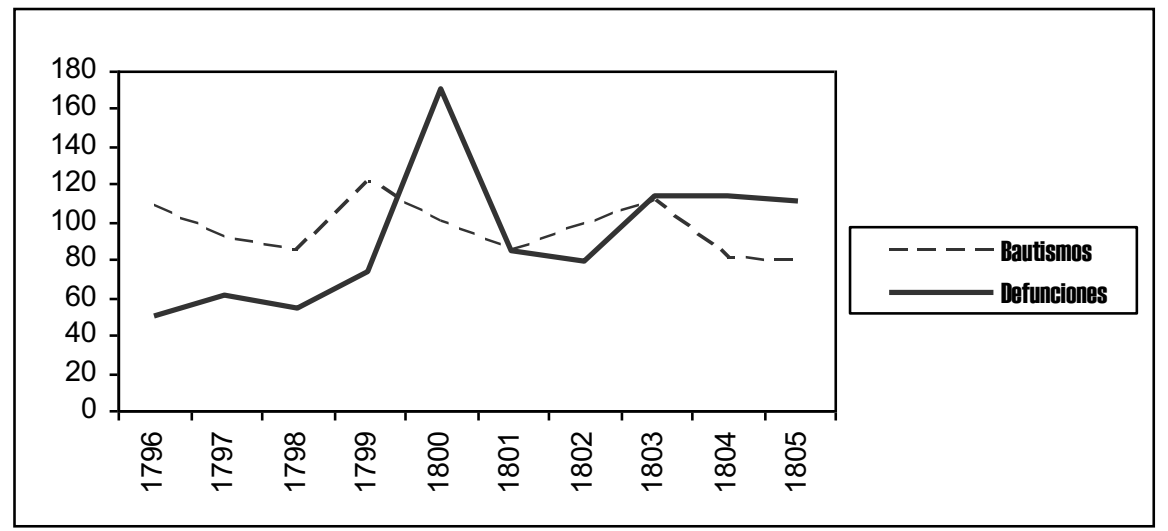

Fuente: A.P.LC., Libros de Bautismos y Entierros. Elaboración propia

Una circunstancia que forzaría a las autoridades neopoblacionales a solicitar que se acordonase el casco urbano de La Carlota para impedir su propagación. Es decir, se procedió a cortar toda comunicación entre las áreas contagiadas y las sanas por medio de un cordón de tropas ${ }^{31}$. No obstante, la efectividad de esta medida en los primeros momentos parece que no fue la que cabría esperar ${ }^{32}$, ya que se ausentarían del referido casco más de doscientos individuos, incluidas sus dos máximas autoridades: el subdelegado y el alcalde mayor ${ }^{33}$. Es más, a finales de octubre sabemos que solo quedaban unos doscientos individuos en el interior del cordón, no tanto por la reducción de individuos a consecuencia de las defunciones como sí por haber huido del núcleo urbano más de cuatrocientas personas hasta entonces $^{34}$. Del mismo modo, otra prueba más de los fallos de este cordón podríamos hallarla en el hecho de que, en alguna ocasión, cuando se denegaba el permiso correspondiente para introducir los cadáveres de los que fallecían fuera del cordón para su entierro en el cementerio

\footnotetext{
31 No disponemos de datos concretos acerca del modo de establecimiento y características de este cordón sanitario, salvo que se situaba a media legua de la población. Ahora bien, pensamos que se seguirían en sus puntos fundamentales las pautas que por aquel entonces fueron habituales en otras entidades poblacionales. Éste había de situarse, como máximo, “á una legua de toda la circunferencia contagiada, pues qualquiera otro mayor radio ó distancia constituirá inútil totalmente el cordón, y no es posible que haya tropas ni paisanos que lo formen tan cerrado como conviene". Y, una vez establecido, lógicamente, había que "enviar toda especie de socorros á los infelices atacados, en el concepto de que en semejantes apuros de todo carecen" (Reflexiones acerca de la epidemia que reyna en Cádiry medios de atajar los estragos de una peste, Córdoba, 1800, s.i., p. 10).

32 Un hecho que contrasta con la rigidez con la que se establecían estos cordones sanitarios. Básicamente, se marcaban dos líneas, justo en el punto medio de la distancia entre las tropas y los lugares contagiados, para que una sirviera de límite a los bloqueados y otra a los del cordón, distantes entre sí cien pasos. Todo el que se adelantase más de cincuenta pasos, fuera de la parte que fuera, sería pasado por las armas. Además, solo se permitía la comunicación entre ambos sectores en las barreras destinadas a ello (Reflexiones acerca de la epidemia..., p. 13).

33 A.P.LC., Libro $2^{\circ}$ de Entierros, f. $1 \mathrm{r}$.

34 A.H.N., Fondos Contemporáneos, Gobernación, leg. 330, exp. 9.
} 
(que había quedado dentro), algunos colonos lograron su objetivo cruzándolo sin que se les viese $^{35}$. Un hecho que, sin duda, estuvo incentivado por la concesión de algunos permisos con este mismo propósito a otros vecinos de la localidad ${ }^{36}$. Obviamente, todos aspiraban a enterrar a sus difuntos en tierra sagrada e hicieron lo posible para lograrlo; más aún si la prohibición no era absoluta.

Ahora bien, a pesar de lo expuesto, sería injusto no reconocer la utilidad de esta medida, ciertamente la única verdaderamente eficaz para la contención de los brotes epidémicos en la época. Salvando las disfunciones mencionadas, el cordón actuó con rigor para impedir la propagación de la enfermedad; algo que se observaba con temor por parte de todas las localidades del entorno, que constituyeron Juntas Municipales de Sanidad y fijaron férreas disposiciones para proteger la salud de sus habitantes. Veamos, por ejemplo, lo ocurrido en tres de ellas: Écija, Córdoba y Santaella.

En septiembre de 1800, como primera medida, la ciudad sevillana de Écija se cerró con tapias, dejando solo cinco puertas con una guardia apostada; siendo necesario para cruzarlas el uso de pasaportes. Sin embargo, ante el agravamiento de la situación, a fines de ese mismo mes se decide establecer también un cordón sanitario ${ }^{37}$. Las autoridades municipales de Córdoba, ante la presencia de la enfermedad en La Carlota, distante apenas treinta kilómetros, se reunieron en Junta de Sanidad el 30 de septiembre con objeto de decretar una serie de disposiciones entre las que destacaron el corte de comunicaciones, tanto de personas como de géneros, con áreas infectadas, el establecimiento de lazaretos para las personas sanas y el de un hospital para los enfermos procedentes de dichas zonas. Igualmente, también aquí se hizo obligatorio el uso de pasaporte hasta el restablecimiento de comunicaciones en marzo del año siguiente ${ }^{38}$.

La epidemia de 1800 tampoco alcanzó la villa de Santaella, situada al sur de La Carlota. A pesar de ello, traemos aquí su caso a colación por el hecho de que alguien informó a la

\footnotetext{
35 A.P.LC., Libro $2^{\circ}$ de Entierros, ff. 11v-12r. A modo de ejemplo, podemos ofrecer la siguiente partida: "En La Carlota a diez y siete de diciembre de mil y ochocientos se dio sepultura en el camposanto de ella a un mozo que murió en La Fuencubierta, y entró por el cordón sin ser sentido a las diez de la noche, llamado Manuel Maqueda, hijo de Juan Maqueda y de Lucía Rodríguez [...]".

36 A.P.LC., Libro $2^{\circ}$ de Entierros, f. 12r. "En La Carlota a diez y seis de enero de mil ochocientos y uno se dio sepultura en el camposanto de ella al cadáver de Jorge Suflo, marido que fue de Mariana Tirlere, murió fuera del cordón pero la tropa dio su permiso para enterrarlo en sagrado".

37 MARTÍN OJEDA, Marina: “Epidemias de fiebre amarilla en Écija. Años 1800 y 1804”, en Actas del V Congreso de Historia «Écija en la Edad Contemporánea», Écija, Ayuntamiento de Écija, 2000, p. 319. Las tropas de este cordón no fueron retiradas, al igual que en La Carlota, hasta principios de marzo de 1801; no obstante, la vigilancia en las puertas y las tapias no cesó hasta finales de dicho año.

38 ARJONA CASTRO, Antonio: Op. cit., pp. 27-32. Gracias a estas medidas la ciudad de Córdoba no resultó afectada por la epidemia de fiebre amarilla, un hecho que ésta agradecería el 18 de junio de 1801 a través de un solemne Te Deum.
} 
Junta de Sanidad de Córdoba de que esta localidad estaba infectada, lo que implicó que inmediatamente se impidiese cualquier movimiento de su población. No obstante, la Junta Municipal de Santaella, víctima a su parecer de un acto malintencionado, escribirá a la Córdoba, con fecha de 7 de diciembre, en estos términos: "estamos en salud, y que no hay epidemia pestilencial, y que lo asegurado a esa Junta [procede de] un falso calumniador y digno del más severo castigo". Comprobada la falsedad de la información, se ordenará restablecer la comunicación cinco días más tarde ${ }^{39}$. Desconocemos las razones por las que las autoridades de Santaella estaban convencidas de que había sido un rumor malintencionado, ya que parecen conocer la identidad del sujeto que lo había propagado al afirmar que debería ser castigado, pero no podemos dejar de plantear que ante la difusión de este tipo de epidemias, aunque algunos pudieran aprovechar para obtener rédito personal, el temor de la población y la falta de información llevaría a sospechar fácilmente de la difusión de la enfermedad ante casos de individuos con síntomas similares a los padecidos por los afectados por la fiebre amarilla.

\subsection{La incidencia demográfica de la epidemia en La Carlota}

La correcta evaluación del impacto que esta epidemia tuvo en La Carlota se ve limitada por la información incluida en las fuentes disponibles. Circunstancia que, aunque nos impida conocer este episodio con el nivel de detalle que nos gustaría, nos deja al menos acceder a algunas conclusiones generales de manera aproximada. En primer lugar, las cifras de población más cercanas a 1800 que hasta ahora hemos localizado son las de $1792^{40}$ y las de $1805^{41}$; sin duda, algo alejadas como para poder establecer juicios seguros. Por lo que, grosso modo, basándonos en ellas y en el ciclo de nacimientos y defunciones, podemos suponer que justo antes de comenzar la epidemia esta colonia contaría con unos 450 vecinos, aproximadamente unos 1.800 habitantes. Asimismo, tampoco disponemos de datos del número de enfermos y restablecidos; tan solo las de óbitos, los que sospechamos que pudieron ser inferiores al número real de fallecimientos. Un hecho nada extraño si consideramos que la parroquia y el cementerio de la colonia quedaron en el interior del cordón de tropas, lo que dificultaba el registro de las muertes ocurridos fuera de él,

\footnotetext{
39 MOYANO LLAMAS, Pablo: "La epidemia de 1800 en Santaella", en Crónica de Córdoba y sus pueblos V, Córdoba, Asociación Provincial Cordobesa de Cronistas Oficiales, 1998, p. 350.

${ }^{40}$ Biblioteca Nacional de España (en adelante B.N.E.), ms. 7.294, f. 418r. En 1792 La Carlota tenía 410 vecinos, de los que 134 vivían en el casco urbano homónimo.

41 A.P.LC., Padrón de 1805. Para la colonia de La Carlota se constatan en este año un total de 478 vecinos.
} 
especialmente en el caso de los párvulos. Es más, estos momentos tan difíciles también podrían haber llevado a un posible subregistro en el propio casco urbano ${ }^{42}$.

Basamos esta hipótesis del subregistro en la distribución de óbitos en La Carlota por sexos y edades. Frente a otros ámbitos geográficos, como por ejemplo Cádiz ${ }^{43}$, en La Carlota no se percibe una especial incidencia de la enfermedad en los niños. Tanto es así que casi no existen partidas de entierro de párvulos en los meses finales de 1800 y los primeros del año siguiente. Sí se observa, en cambio, como en aquellos, una mayor mortalidad entre los varones que entre las mujeres (Gráficos 2 y 3).

\section{Gráfico 2}

\section{Evolución de la mortalidad por sexo. La Carlota (septiembre 1800-marzo 1801)}

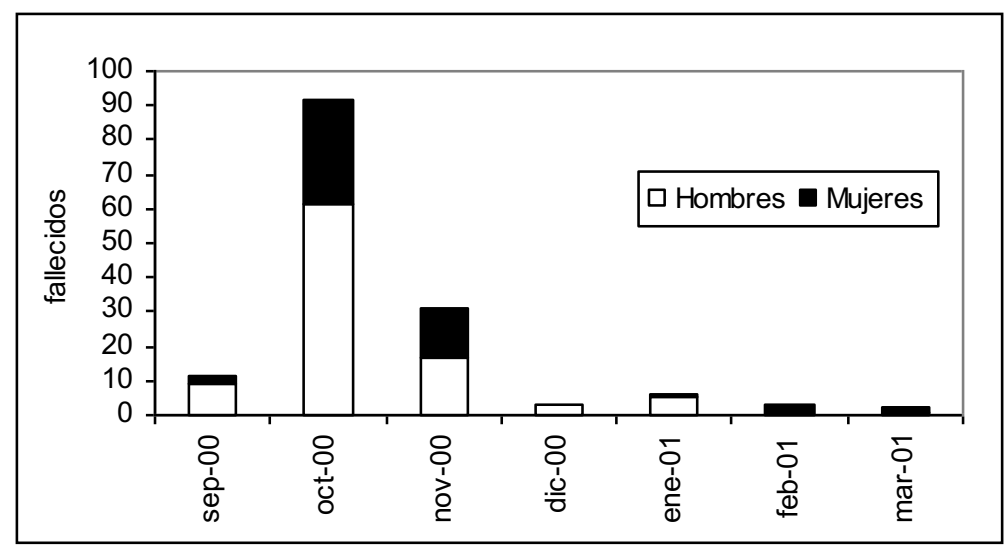

Fuente: A.P.LC., Libros de Entierros. Elaboración propia

Disponemos, ciertamente, de varias fuentes que nos informan sobre el número de vidas que la epidemia de 1800 se cobró en La Carlota. Sin embargo, como ya hemos afirmado, dudamos de que se ajusten a la realidad. El capellán fray Juan Romero de La Cruz indica que fallecieron más de 145 individuos $^{44}$, mientras que Alfonso de María nos habla de 147 víctimas mortales ${ }^{45}$. Unas cifras casi coincidentes y que casualmente casi coinciden con el recuento

\footnotetext{
42 A.P.LC., Libro $2^{\circ}$ de Entierros. Las páginas disponibles para anotar partidas en el primer tomo se agotaron justo al inicio de la epidemia. La uniformidad y detallismo con el que se redactaron las primeras partidas del segundo libro de Entierros de La Carlota, sobre todo las comprendidas entre septiembre de 1800 y enero de 1801, nos hacen pensar que su inscripción fue también posterior a la retirada del cordón de tropas; quizá hasta entonces en la parroquia solo se llevara un borrador de los entierros que se produjeron en el cementerio de La Carlota. De este modo podría comprenderse que todas las partidas inscritas en este Libro $2^{\circ}$ de Entierros tengan un perfecto orden cronológico, un hecho del todo imposible sin ese registro previo, ya que se insertan también partidas de entierros que se produjeron fuera del cordón y que, lógicamente, fueron desconocidos hasta tiempo después por el sector eclesiástico.

${ }^{43}$ IGLESIAS RODRÍGUEZ, Juan José, Op. cit., p. 73.

${ }^{44}$ A.P.LC., Libro $2^{\circ}$ de Entierros, f. $1 \mathrm{r}$.

${ }^{45}$ MARÍA, Alfonso de: Memoria sobre la Epidemia de Andalucía el Año de 1800 al 819, Cádiz, 1820; citamos por
} 
del número de partidas de entierros de La Carlota, registradas en los libros de su parroquia, entre el 2 de septiembre de 1800 y el 7 de marzo del año siguiente; que ascienden a 146. Por tanto, quizá el número de fallecidos fue algo superior, situando el porcentaje de óbitos en esta colonia por encima del $8 \%$ de la población total ${ }^{46}$.

\section{Gráfico 3}

Evolución de la mortalidad por grupos de edad. La Carlota (septiembre 1800-marzo 1801)

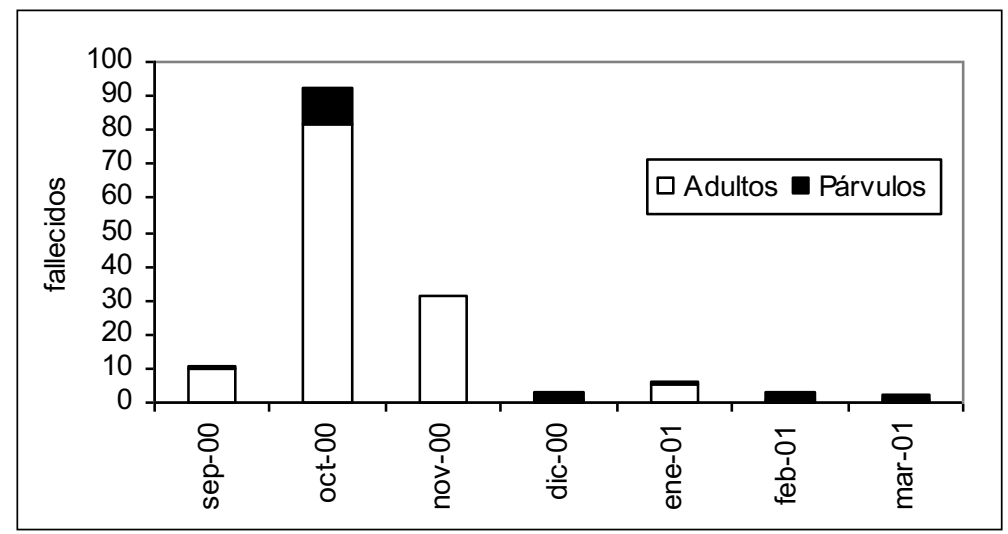

Fuente: A.P.LC., Libros de Entierros. Elaboración propia

Al parecer, la epidemia alcanzó su mayor intensidad a finales de octubre (véase el Gráfico 4), momento en el que ya habían tenido que cerrarse nueve casas en el casco urbano de La Carlota por haber muerto todos sus ocupantes. En algunos casos prácticamente desaparecieron familias enteras (véase el Cuadro 1), lo que incrementó el temor popular a esta epidemia ${ }^{47}$. Tanto es así que un testimonio de 1802 nos indica que el terror con el que los viajeros miraron durante mucho tiempo la Real Posada de La Carlota, foco del brote epidémico, fue tal que pocos estaban dispuestos a alojarse en ella ${ }^{48}$.

IGLESIAS RODRÍGUEZ, Juan José: Op. cit., p. 51. Esta cifra de óbitos haría que La Carlota ocupase el puesto número veintiuno del ranking de localidades con mayor mortalidad. A pesar de ser la localidad más alejada del foco originario (Cádiz), la epidemia se mostró aquí con una especial letalidad. Téngase en cuenta que para localizar otras poblaciones con un número superior de finados tenemos que ir a El Arahal (con 1.800) y a Los Palacios y Villafranca (con 192).

46 Aunque desconocemos la cifra de habitantes del casco urbano de La Carlota para el año 1800, por referencias indirectas (huyen más de doscientos y fallecen, al menos, centenar y medio) sabemos que era superior a seiscientos habitantes. Un número bastante cercano a la realidad tal vez sería el de unos ochocientos individuos al comenzar el mes de septiembre. Ello supondría un porcentaje de defunciones superior al 16\% para la zona comprendida dentro del cordón.

47 Todavía a comienzos del siglo XX, algunos testimonios orales de vecinos de La Carlota se hacían eco del fuerte impacto que tuvo este episodio epidémico en dicha comunidad.

48 Archivo Notarial de Posadas (en adelante A.N.PO.), La Carlota, Escribano Juan Vázquez, prot. 952, año 1802, s.f.). El escribano afirma el 4 de octubre de 1802 que "con motivo de la enfermedad contagiosa que en el año 
Su intensidad no aminoraría hasta mediados de noviembre (Gráfico 5), cuando el uso de abundantes limonadas y refrescos y, en menor medida, de la quina, empezaron a mostrar buenos resultados ${ }^{49}$. Además, tampoco puede descartarse que la entrada del otoño facilitara la desaparición de las condiciones ambientales que facilitaban la propagación de la enfermedad.

\section{Cuadro 1}

Efectos de la fiebre amarilla en una familia de La Carlota (1800)

\begin{tabular}{|l|l|l|l|l|}
\hline Abuelos & Nacimiento & Matrimonio & Defunción \\
\hline \multirow{2}{*}{ Paternos } & $\begin{array}{l}\text { Alonso Tomás Pintado } \\
\text { María de Varo }\end{array}$ & $?$ & $?$ & $11 / 8 / 1785$ \\
\hline Maternos & $\begin{array}{l}\text { Cristóbal S. Hidalgo } \\
\text { Isabel Jurado }\end{array}$ & $?$ & $?$ & $16 / 7 / 1795$ \\
\hline Padres & $?$ & $?$ & $\mathbf{2 2 / 9 / 1 8 0 0}$ \\
\hline $\begin{array}{l}\text { Juan Miguel Blas Pintado } \\
\text { María Antonia Delgado }\end{array}$ & Nacimiento & Matrimonio & Defunción \\
\hline Hijos & $?$ & $21 / 6 / 1784$ & $\mathbf{5 / 1 0 / 1 8 0 0}$ \\
\hline $\begin{array}{l}\text { 1. Isabel } \\
\text { 2. María }\end{array}$ & Nacimiento & $\mathbf{D e f u n c i o ́ n ~ y ~ e d a d ~}$ & Observaciones \\
\hline
\end{tabular}

Fuente: A.P.LC., Libros de Bautismos, Matrimonios y Entierros. Elaboración propia

En cualquier caso, fuera cual fuera la cifra exacta de finados, su elevado número hizo necesario ampliar el cementerio de la colonia de La Carlota ${ }^{50}$. Una ampliación provisional y de reducidas dimensiones en un primer momento, como lo prueba que en 1803 ya no quedara espacio para nuevos sepelios ${ }^{51}$, y que ese mismo año se completaría con una intervención

próximo pasado de mil ochocientos se propagó en esta dicha capital, mayormente en su posada fonda, en que por haber fallecido su arrendador Juan Irigoyen, y cuasi todos los sirvientes de dicha fonda, quedó cerrada, de cuya manera permaneció mucho tiempo después por estar cortada la comunicación, y posteriormente por el horror con que los pasajeros miraban dicho parador". Aunque se logró su arriendo el 14 de marzo de 1801, el beneficiario consiguió una revisión del contrato por real orden de 4 de febrero de 1802, pues el miedo de la gente a entrar en el inmueble afectaba notablemente a la previsión de ingresos con los que satisfacer las cuotas fijadas.

49 A.H.N., Fondos Contemporáneos, Gobernación, leg. 330, exp. 9. En este sentido se pronuncia el médico y cirujano de La Carlota, Dionisio María de Velasco.

${ }^{50}$ RAMÍREZ Y LAS CASAS-DEZA, Luis María: Corografía histórico-estadística de la provincia y obispado de Córdoba, Córdoba, Diputación Provincial de Córdoba, 1986, vol. II, p. 245. Estas obras de ampliación del cementerio habrían tenido lugar, según nos indica Ramírez y las Casas-Deza, en 1800; sin embargo, consideramos que probablemente entonces solo se delimitó provisionalmente una nueva área de enterramiento. El levantamiento de tapias y estructuras definitivas es más lógico pensar que tuviera lugar a partir de marzo de 1801, una vez retirado el cordón sanitario.

51 Archivo General del Obispado de Córdoba (en adelante A.G.O.C.), Despachos Ordinarios, caja 7117, exp. 1. El capellán fray Juan Romero de la Cruz escribe al subdelegado de La Carlota en estos términos el 3 de julio de 
que duplicó el espacio disponible. Una intervención que garantizaría espacio suficiente para atender a las necesidades de la localidad hasta la ampliación de 1950.

\section{Gráfico 4}

Evolución mensual de la mortalidad. La Carlota, 1798-1803

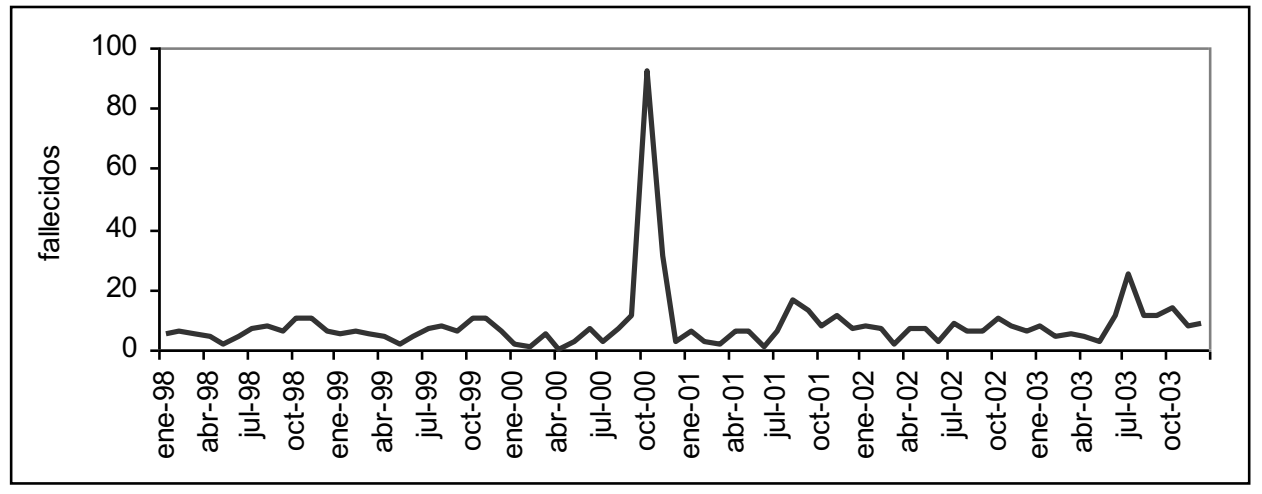

Fuente: A.P.LC., Libros $1^{\circ}$ y $2^{\circ}$ de Entierros. Elaboración propia

\section{Gráfico 5}

Evolución semanal de la mortalidad. La Carlota, 2/9/1800-7/3/1801

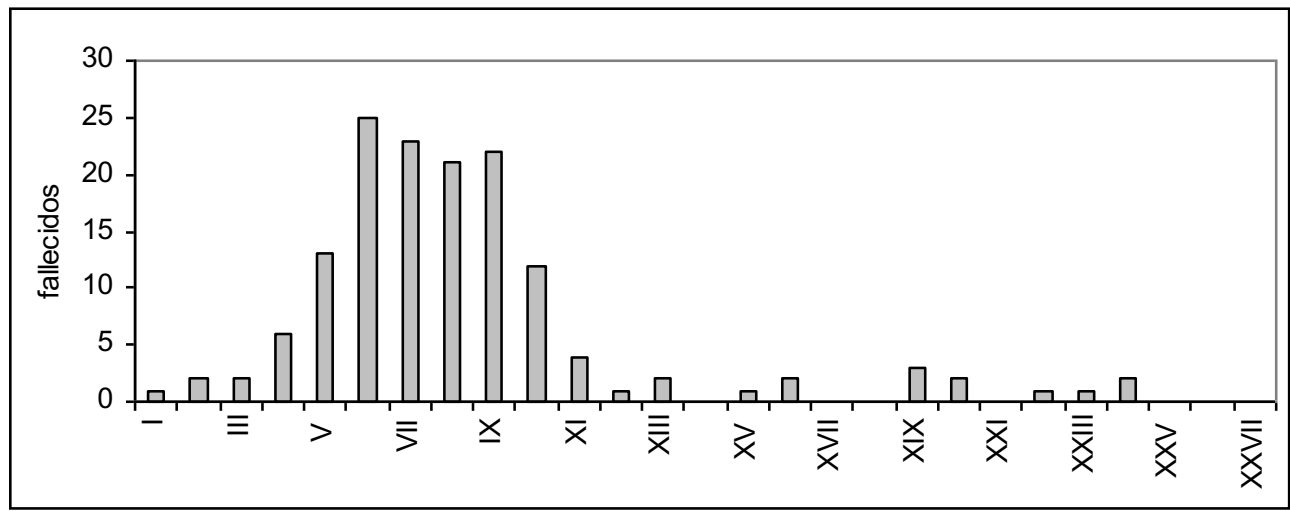

Fuente: A.P.LC., Libros $1^{\circ}$ y $2^{\circ}$ de Entierros. Elaboración propia

1803: "He visto el camposanto nuevo con toda reflexión, y no hallo que haya espacio de tierra en el que se puedan sepultar cuatro muertos, he preguntado a el enterrador y examinado a el maestro mayor de albañil Juan Diego de Aguilar, comisionado por vuestra señoría para que lo registre, y los dos contestes me dicen estar lleno; el camposanto antiguo y contiguo quedó imposibilitado para muchos años por los difuntos que en él se enterraron el año de 800 de la epidemia general que padeció esta población quedando ocupado de dichos difuntos todo su terreno como se me ha respondido por el sacristán mayor Antonio de Luque, y por otros varios. En la iglesia está prohibido por reales órdenes y de los señores obispos, por lo que se hace forzoso que, a la mayor brevedad, como lo exige la necesidad, y estación del tiempo se emprenda la obra de otro camposanto, lo que participo a vuestra señoría para su gobierno". La respuesta de Joaquín Cadiou no se hizo esperar, dando órdenes para que se abrieran los cimientos de las tapias inmediatamente. 


\subsection{Los efectos sociopolíticos: el comportamiento ante la enfermedad}

Una vez analizado, en sus líneas generales, el impacto demográfico que tuvo la epidemia en La Carlota, sería conveniente abordar cuáles fueron sus efectos a nivel sociopolítico. Situaciones tan adversas como la aparición de enfermedades epidémicas suponían en la Edad Moderna un acontecimiento que sobrecogía intensamente a la sociedad dentro de la cual hacían acto de presencia. Era precisamente entonces cuando se mostraban con mayor evidencia las tensiones y diferencias sociales y cuando mejor podía observarse el talante de los gobernantes y el grado de obediencia de los gobernados. Ni que decir tiene que La Carlota no constituyó, en este sentido, una excepción a lo que fueron las respuestas verificadas normalmente en las entidades poblacionales donde se daba un brote epidémico. Tanto las autoridades, valiéndose para ello de los empleos que desempeñaban, como los habitantes de las poblaciones tendieron a huir de la zona infectada ${ }^{52}$. En el apartado anterior ya dimos cuenta del temor que esta epidemia de 1800 generó durante mucho tiempo no solo entre los vecinos sino también entre los viajeros que la transitaban a través del camino real. El mejor modo para comprender lo ocurrido en esta colonia durante la epidemia y los meses posteriores a su desaparición no es otro que el de narrar cronológicamente lo acontecido, solo así podremos hacernos una idea aproximada de lo tensa y compleja que fue la situación ${ }^{53}$.

Desde la constatación del primer caso de fiebre amarilla en la capital de las Nuevas Poblaciones de Andalucía a principios de septiembre de 1800 hasta finales de dicho mes solo se registraron muertes esporádicas, tal vez porque la enfermedad estaba en su periodo de incubación. La epidemia parece que solo comenzó a mostrarse claramente a partir del día 30 de septiembre, según se deduce de la correspondencia de las autoridades neopoblacionales. Un hecho que llevó al alcalde mayor de La Carlota, Antonio Basilio de Acosta, a abandonar la localidad, en compañía de su familia, el día 2 de octubre ${ }^{54}$. Pero estos no serían los únicos, otros empleados de la administración neopoblacional procedieron de igual modo en esos días ${ }^{55}$; destacando, sin duda, el caso de la máxima autoridad, el subdelegado Joaquín Cadiou

\footnotetext{
52 "En el año de 1800, día 2 de septiembre, dieron principio en esta población las enfermedades contagiosas que duraron hasta el día 16 de noviembre del mismo año, en cuyo tiempo estuvo acordonado todo el pueblo sin dejar entrar ni salir a ningún colono; se ausentaron más de 200 personas" (A.P.LC., Libro $2^{\circ}$ de Entierros, ff. $1 \mathrm{r})$.

53 Todos los datos que se consignan en los párrafos siguientes de este apartado, salvo que se indique lo contrario, han sido tomados de: A.H.N., Fondos Contemporáneos, Gobernación, leg. 330, exp. 9.

${ }^{54}$ Este hecho le supondría el ser cesado de su cargo, el cual se concedió a Ignacio Pablo Sandino de Castro. Así se pronuncia un testimonio de la época: "El alcalde mayor fue depuesto de su empleo y en su lugar vino don Ignacio Pablo Sandino" (A.P.LC., Libro $2^{\circ}$ de Entierros, f. 1r).

${ }^{55}$ Entre ellos podemos mencionar a Juan Vázquez, escribano de la Subdelegación y del Juzgado de la Alcaldía
} 
Praet, que dejó el casco urbano el día cinco de dicho mes $^{56}$.

Unos abandonos que dieron lugar a un vacío de poder y hasta de asistencia médica en la localidad, pues ya se había establecido el cordón sanitario. Del gobierno del casco urbano hubo de encargarse provisionalmente el contador, Agustín de Murua, siendo auxiliado en esta labor por el oficial primero de dicha Contaduría, Mariano Fernández. A ellos correspondió, qué duda cabe, hacer frente a los momentos más críticos de la epidemia. En lo que respecta a la atención médica, no nos consta que quedase en el núcleo urbano ningún médico o cirujano que pudiera ocuparse de los enfermos; por tanto, es de suponer que fueron sus familiares los que los cuidaron y, en caso de no tenerlos, es lógico pensar que las autoridades gestionaran su cuidado. La existencia del cordón no impedía que, siempre que se cumpliesen los protocolos establecidos, se facilitaran indicaciones médicas, productos y remedios desde el exterior; un hecho que, aunque alejaba el peligro de una completa desatención, en modo alguno podía suplir la presencia de un galeno junto a los enfermos. Del mismo modo, la existencia de abundante cereal tanto en el pósito de labradores como en los pósitos de diezmos ${ }^{57}$ permitía que las autoridades interinas pudieran garantizar un abastecimiento mínimo a la población acordonada que, en caso necesario, podría completarse con productos facilitados desde el exterior. En una Intendencia con un sistema de gobierno foral marcadamente paternalista, la existencia de un episodio de desabastecimiento o carestía habría manchado irremediablemente la hoja de servicios de sus gobernantes, especialmente de los que habían abandonado el casco urbano, por lo que puede descartarse su aparición durante esta epidemia. Además, el silencio sobre este punto en toda la correspondencia oficial avala aún más hipótesis.

La explicación que Cadiou ofreció para justificar su marcha no llegó a convencer a otros miembros de la administración. En términos generales afirmaba que el día cuatro de octubre, después de dos días sin dormir ni descansar, había comenzado a sentirse mal, circunstancia por la cual los médicos le habían aconsejado abandonar la población; lo que

\footnotetext{
Mayor; a Dionisio María de Velasco, médico y cirujano de La Carlota; o a José María Custodio, escribano del juzgado de la Alcaldía Mayor.

56 Joaquín Cadiou, como subdelegado de las Nuevas Poblaciones de Andalucía, gobernaba este partido territorial de la Intendencia de las Nuevas Poblaciones de Sierra Morena y Andalucía (que comprendía las colonias de La Carlota, Fuente Palmera, La Luisiana y San Sebastián de los Ballesteros) bajo las ordenes del intendente. Era, por tanto, la máxima autoridad en ellas. El alcalde mayor tenía limitadas sus competencias a la justicia ordinaria y a asesorar a la Subdelegación en aquellas cuestiones que lo requiriesen.

57 Estos pósitos se ubicaban en la misma manzana de la real posada de La Carlota, foco de la epidemia. Las autoridades locales podían disponer de sus granos, especialmente de los correspondientes al diezmo, porque correspondían a la Real Hacienda. En las Nuevas Poblaciones de Sierra Morena y Andalucía, desde su fundación y al ser tierras, en su mayor parte, puestas en cultivo por primera vez, el cobro del diezmo correspondía al rey y no a la Iglesia.
} 
hizo al día siguiente pasando a una casa de campo situada a media legua del casco y junto al cordón ${ }^{58}$. El día siete, según sostenía, habría intentado entrar de nuevo en la población, pero el comandante del cordón ${ }^{59}$ le manifestó que si lo hacía no lo dejaría salir; de ahí que rehusase volver, pues entonces hubiera dejado desatendida no solo la mayor parte de la colonia de La Carlota, sino también a las restantes Nuevas Poblaciones de Andalucía. El 21 de octubre, Cadiou informó por carta a Agustín de Murua que volvería al casco urbano dos días después, pero al oír el mismo argumento de boca del comandante del cordón, prefirió quedarse junto a éste, asistido por el escribano y el alcalde mayor. Murua, que sospechaba que el subdelegado y otros individuos disfrazaban su huída con la excusa de una mayor utilidad fuera del cordón, decidió poner el asunto en conocimiento del Consejo de Castilla; que, con bastante celeridad, acordó el 26 de octubre que Cadiou cesase interinamente hasta que se pudiera dar una resolución definitiva; una noticia que se recibirá en La Carlota cuatro días más tarde. Una resolución definitiva que llegó en forma de real orden de 4 de noviembre en la que se cesó al subdelegado y se nombraba subdelegado interino al propio Agustín de Murua y como contador interino a Mariano Fernández. Dos días más tarde, otra real orden se ocuparía del cese del resto de desertores ${ }^{60}$.

Obviamente, Joaquín Cadiou no estaría dispuesto a aceptar dicha resolución, sobre todo porque venía a manchar gravemente su cursus honorum, pues justificaba su cese en el hecho de haber huido de sus obligaciones. De ahí que marchase inmediatamente a la corte para defenderse. A finales de noviembre lo encontramos ya en San Ildefonso, desde donde remite a Carlos IV un memorial en el que justifica su proceder afirmando que no quiso dejar desatendidos al resto de colonos de las Nuevas Poblaciones de Andalucía en unos momentos tan críticos. Un argumento que, aunque no excusaba su proceder, quizá tuvo gran peso en las decisiones posteriores. Este partido territorial se componía de cuatro feligresías, no existiendo continuidad territorial entre todas ellas, en las que precisamente se estarían desarrollando entonces las labores de siembra; las cuales tenían una importancia enorme en estas colonias toda vez que, como indicábamos, el diezmo correspondía a la Real Hacienda.

\footnotetext{
${ }^{58}$ Se trataba, concretamente, de la quinta casa dispersa situada en dirección a Écija; en la suerte del “cordobés". Cadiou la elegiría "por estar en el mismo arrecife, inmediata al sitio designado para mercado público de unos y otros vecinos".

${ }^{59}$ Nos consta que este comandante era el marqués de la Solana. En otro orden de cosas, Agustín de Murua se quejará en los primeros días de octubre del enorme celo mostrado por este comandante, que se negaba incluso a recibir los pliegos en los que informaba de la situación en la zona contagiada. Un hecho que contrasta enormemente con el elevado número de colonos que lograron huir a través del cordón en estos mismos días.

${ }^{60}$ Entre ellos se situaban, por ejemplo, el tesorero Joaquín de Furundarena y el escribano de la Alcaldía Mayor, José María Custodio.
} 
Se inició a partir de ese momento un periodo de enorme tensión política a nivel local. Una hipótesis que viene avalada por hechos tales como que el intendente de las Nuevas Poblaciones de Sierra Morena y Andalucía, Tomás José González Carvajal, procediese el 14 de noviembre a proponer una serie de candidatos para cubrir las vacantes dejadas por las ya mencionadas reales órdenes de octubre, y que al día siguiente cambiase completamente de parecer con respecto a Joaquín Cadiou. Mientras el día 14 aceptaba su cese, proponiendo hasta quién habría de sucederle, y el 15 afirmaba no ver tan clara la vacante ${ }^{61}$. Por otro lado, la descoordinación reinante y la falta de medios dentro del área acordonada permitirían que, aprovechando un menor vigor de la epidemia, se produjera un intento frustrado de robo de los caudales de la Real Hacienda en la noche del 21 al 22 de ese mismo mes.

Finalmente, tras un invierno bastante frío y riguroso ${ }^{62}$, "se quitó el cordón de las tropas que circundaba el pueblo el día 7 de marzo de 1801 a las tres de la tarde, y se hizo salva por los del pueblo, y hubo repique en acción de gracias, y al día siguiente misa con sacramento y procesión por las calles con el Señor de la Misericordia y la Virgen del Rosario" ${ }^{63}$. La epidemia había desaparecido por completo ${ }^{64}$, aunque ello no significó una inmediata vuelta a la normalidad en los temas de gobierno. Las interinidades de aquellos que se habían hecho cargo provisionalmente de la Subdelegación y de la Contaduría se prolongaron durante dos años, concluyendo solo el 14 de agosto de 1802; fecha en la que Joaquín Cadiou fue repuesto en su antiguo empleo, prueba inequívoca que los argumentos

\footnotetext{
${ }^{61}$ Las vacantes hacen que González Carvajal proponga, el 14 de noviembre, como subdelegado a Agustín de Murua (contador), como contador a Mariano Fernández (oficial $1^{\circ}$ de la Contaduría) y como tesorero a Mariano Castillejo (colegial real de Granada, y ahijado del propio intendente). Sin embargo, al día siguiente defenderá el reintegro a su puesto de Cadiou, del que afirmará que es "un pobre anciano que, consternado a la vista de una calamidad nueva y desconocida para él, falto de consejo, arrastrado del ejemplo del asesor [Antonio Basilio de Acosta], que pudiese habérselo dado, instado o importunado, como es natural, de su familia, resolvió salir sin saber tal vez lo que resolvería y esperando que podía volver". Es probable que el intendente recibiera alguna carta de Cadiou el día 14 descargando toda la responsabilidad en el alcalde mayor, la cual quizá hizo cambiar de opinión al intendente.

${ }^{62}$ Este detalle no es en absoluto gratuito, pues hemos de tener en cuenta que los individuos que permanecieron dentro del cordón, debido a su aislamiento forzoso, carecerían no solo de muchos de los alimentos necesarios sino incluso de madera para hacer frente al frío. Por otro lado, estas bajas temperaturas dañaron gravemente las arboledas que lograron sobrevivir a las talas que las tropas del cordón realizaban para mantener encendidos los necesarios fuegos. Por último, no podemos dejar de mencionar los efectos perjudiciales que sobre los campos cultivados tuvieron el paso de arrieros y carruajes que habían de rodear a su paso el casco urbano.

${ }^{63}$ A.P.LC., Libro $2^{\circ}$ de Entierros, f. $1 \mathrm{r}$.

${ }^{64}$ En julio de 1801 la ciudad de Écija difundirá la noticia de que en La Carlota había vuelto la epidemia, para lo cual exhibirá incluso certificados de sus médicos en este sentido. De ahí que obligase a todos los forasteros, especialmente a los colonos de La Carlota, a abandonar dicha ciudad; imponiendo una multa de 500 ducados a quien los acogiese. Desde La Carlota se responderá que se trata solo de algunas tercianas que se curan con refrescos y, las más fuertes, con quina; atribuyendo este desproporcionado proceder a la "antigua rivalidad [de Écija] contra los naturales de [estas] Poblaciones". Ciertamente se reconoce que desde La Carlota van enfermos a curarse a dicha ciudad, pero también que hay otras personas que, "estado buenas, [van] a bañarse en aquel río [Genil]"; este es el caso, por ejemplo, de Nicolasa Rosales, cuñada del subdelegado Joaquín Cadiou.
} 
que esgrimía desde 1800 acerca de su salida de La Carlota durante la epidemia habían sido finalmente aceptados como válidos, total o parcialmente, por el gobierno ${ }^{65}$.

\section{Conclusiones}

Una vez realizado un recorrido por los efectos demográficos y sociopolíticos de la epidemia de fiebre amarilla de 1800 en La Carlota, es el momento de ofrecer las principales conclusiones alcanzadas en este trabajo. La aparición de la enfermedad, de manera sorpresiva pues las localidades del entorno inmediato no resultaron afectadas, estuvo ligada a su emplazamiento junto al camino real que unía Madrid con Cádiz y a la existencia de una real posada que daba alojamiento a los viajeros que lo transitaban. Uno de ellos actuaría como vector de la enfermedad, transmitiéndola al personal de dicho establecimiento, desde donde se propagó por todo el casco urbano.

Una cifra no inferior a casi centenar y medio de individuos fallecería en la localidad desde la declaración de la epidemia a inicios de septiembre de 1800 hasta la retirada del cordón de tropas en marzo de 1801, concentrándose el mayor pico de mortalidad en el mes de octubre pues los remedios aplicados, especialmente los preventivos dado el escaso nivel de eficacia de la medicina de la época del contagio, comenzaron a mostrar su utilidad a partir de noviembre. Además, la propia dinámica de propia epidemia, que ya había afectado a la mayor parte de la población receptiva provocando su inmunización, así como el cese del óptimo climatológico que había favorecido su difusión a medida que avanzó el otoño y disminuyeron las temperaturas, influirían determinantemente para dar fin a esta fase.

La circunstancia de haber quedado la parroquia y el cementerio dentro del cordón nos hace sospechar que pudo haber lagunas en los registros eclesiásticos en entierros. Todo apunta a que buena parte de las actas de entierro se llevaron en registros provisionales, siendo transcritas en el libro una vez retirado el cordón; de ahí que exista la posibilidad de no haberse consignado algunos óbitos, especialmente de párvulos. En cualquier caso, todas las fuentes coinciden en que la epidemia causó un elevado porcentaje de fallecimientos en La Carlota (sobre todo si solo consideramos a los vecinos que residían en el núcleo urbano, que fueron los que quedaron dentro del cordón), evidenciando que la circunstancia de haber sido el punto más alejado de la costa donde se desarrolló entonces la fiebre amarilla no tenía la

\footnotetext{
65 A.H.N., Fondos Contemporáneos, Gobernación, leg. 279, exp. 17. Agustín de Murua, subdelegado interino, había solicitado el 22 de marzo de 1801, no mucho después de quitarse el cordón, la jubilación de su cargo con los 10.600 reales de los que entonces gozaba o, en su defecto "un destino más tranquilo", pero su petición no fue entonces atendida.
} 
menor influencia en la intensidad que podía mostrar si las condiciones ambientales lo favorecían. Una cifra tan elevada que el cementerio de la localidad se llenó, siendo necesario enterrar a los difuntos en un nuevo recinto que posteriormente se incluyó dentro de la ampliación de 1803.

Por otro lado, la aparición de esta epidemia puso sobre la mesa dos realidades habituales en estos casos: de un lado, el terror que generaba entre la población, que no dudaba en huir para no enfermarse (lo que llevaba al establecimiento de cordones de tropas que impidiesen la salida de enfermos y, por tanto, la propagación de la enfermedad); y de otro, la responsabilidad y la fortaleza de los gobernantes y los cargos administrativos para permanecer en sus cargos y gestionar la situación. En el primer caso, nos consta que en La Carlota, al igual que ocurrió en el resto de localidades afectadas por la epidemia, huyeron cientos de vecinos con la esperanza de salvar su vida, y si no se despobló el núcleo urbano fue porque el cordón de tropas lo impidió; mientras que en el segundo, asistimos a la controversia de haber huido el subdelegado, el alcalde mayor y algunos otros empleados, incluido el médico, forzando a que asumieran las funciones de gobierno y administración aquellos que permanecieron en la población. El gobierno central castigó esa forma de proceder desposeyéndolos de sus empleos; no obstante, una vez superada la epidemia, y tras meses de instancias y argumentos que trataban de explicar esa salida del cordón como lo más beneficioso para las labores de gobierno, algunos de los cesados serían reintegrados en sus empleos. De este modo, el subdelegado Joaquín Cadiou recuperaba su empleo y lograba borrar la mancha que aseguraba se había realizado en su trayectoria al servicio del rey.

La epidemia de fiebre amarilla de 1800 significó para la capital de las Nuevas Poblaciones de Andalucía no sólo la pérdida de más de un centenar de sus vecinos, sin contar la desconocida cifra de enfermos que lograron recuperarse, sino también el inicio de un complejo periodo de dos años en el que los principales cargos de gobierno se desempeñaron de manera interina. La normalidad institucional no se recobraría hasta 1802. Más tiempo aún requirió el que el edificio de la Real Posada de La Carlota volviera a disfrutar del éxito que tenía entre los viajeros antes de la epidemia, pues estos durante años la miraron con temor; circunstancia que afectaba directamente a los ingresos que debía generar a su arrendador. 